\title{
Throughput Enhancement of Cognitive Radio Networks through Improved Frame Structure
}

\author{
Rubeena \\ Dept. of Electronics and Communication \\ Chandigarh Engineering College, \\ Mohali, India
}

\begin{abstract}
A cognitive radio (CR) system exploits spectrum bands that are not fully utilized by the licensed users. For the successful deployment of the technology, the biggest challenge is to make network protocols more adaptive to use the available bandwidth effectively. Various medium access protocols (MAC) have been proposed by researchers to manage spectrum sensing and the channel utilization between two kind of users namely licensed primary users and unlicensed secondary users. In this paper, a modified self-scheduling multi-channel cognitive radio MAC protocol based on cooperative communication is proposed to enhance the network throughput by modifying its frame structure. The proposed frame structure also eliminates the sensing throughput tradeoff that was encountered in the conventional scheme. The simulation results show that the proposed scheme significantly outperforms the conventional scheme by increasing the throughput 200Mbps.
\end{abstract}

\section{Index Terms}

Cognitive radio, Primary user, Secondary user, SMC-MAC, FCS, Spectrum Sensing, Throughput.

\section{INTRODUCTION}

The performance of wireless ad hoc networks is mostly limited because of static spectrum allocation by the regulatory bodies. In 2002, the report of FCC revealed that the large portions of assigned spectrum are being used sporadically and spectrum occupancy ranges from $15 \%$ to $85 \%$ in a day. This motivates the concepts of opportunistic spectrum access that allows cognitive radio (CR) networks to opportunistically exploit under-utilized spectrum [1-2]. In November 2008, the FCC ruled that the DTV white spaces can be used by secondary (unlicensed) devices. Each cognitive transmission process requires two essential phases:

\author{
Indu Bala \\ Dept. of Electronics and Communication \\ Chandigarh Engineering College, \\ Mohali, India
}

(i) A spectrum sensing phase, in which a cognitive source attempts to detect an available spectrum hole and

(ii) A data transmission phase, in which secondary data traffic is transmitted to the destination through the detected spectrum hole as discussed in [3-5].

In this paper, the Media Access Control (MAC) protocol is investigated which has significant importance in ad hoc cognitive network. The cognitive MAC should fully utilize the advanced capability provided by cognitive radio to optimize the network performance. Firstly, the cognitive radio should identify the spectrum holes in the licensed user's spectrum band and secondly, utilize them in flexible manner, according to a medium-access control (MAC) scheme. In [611], there are approaches of cognitive MAC have been represented and the main characteristics of cognitive MAC protocol are:

(i) Identify the available spectrum resource through the spectrum sensing

(ii) Decision on the optimal sensing, transmission time

(iii) Proper coordination with other SUs for the spectrum access

In [12], a self-scheduling multi channel cognitive radio MAC (SMC-MAC) protocol has been proposed, in which a secondary user consists of a single transceiver to cooperate with each other. In [12], the fixed channel sensing (FCS) and adaptive channel sensing (ACS) schemes are presented. For FCS, CR system senses the fixed number of channels of licensed channels.

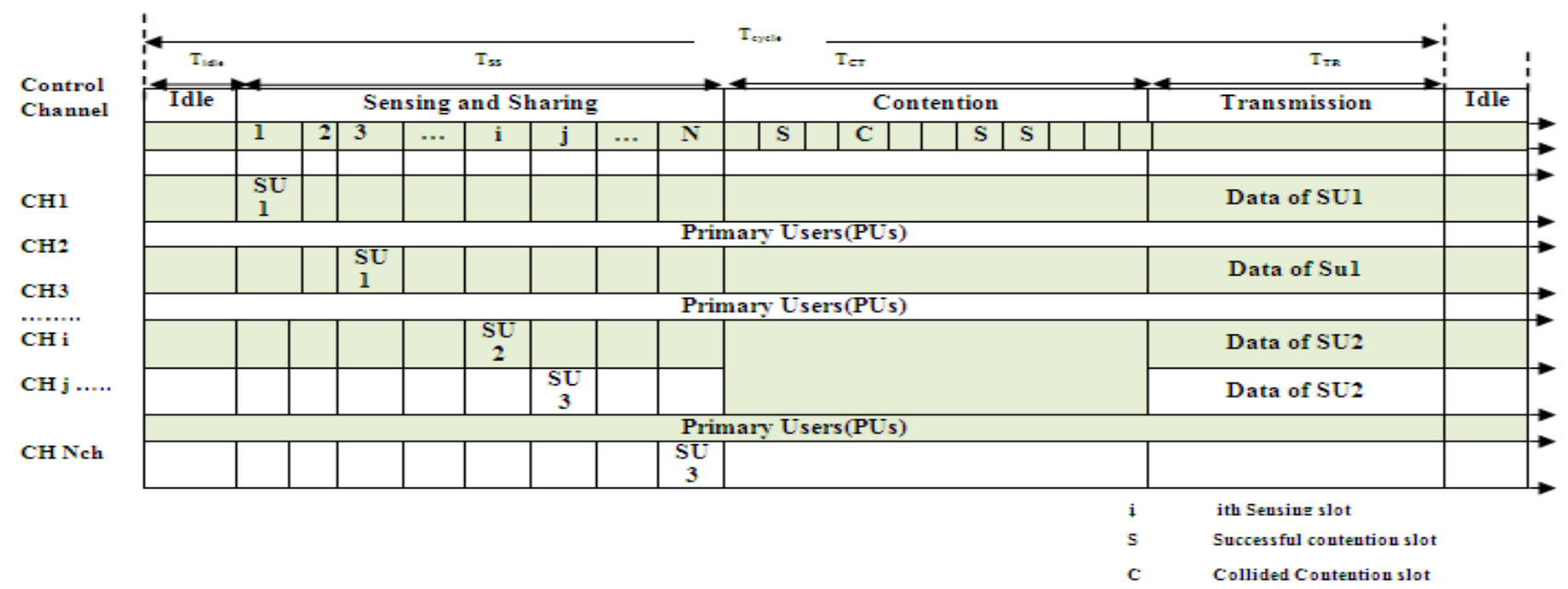

Figure 1: Frame Structure of conventional SMC-MAC Protocol 


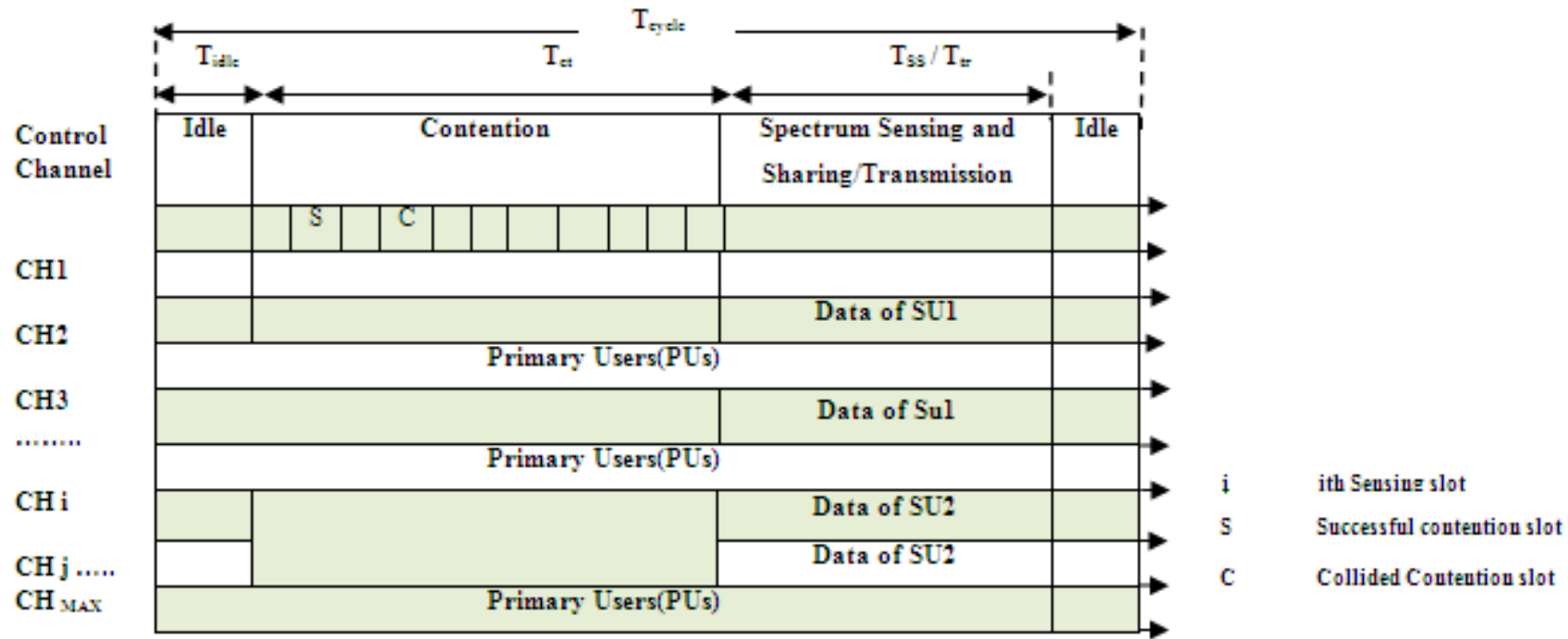

Figure 2: Modified Frame Structure of Proposed SMC-MAC Protocol

\section{CONVENTIONAL FRAME STRUCTURE}

The conventional frame structure of SMC-MAC is presented in fig. 1. In the SMC-MAC protocol secondary users can use one common control channel and up to a number of control channels [12]. A cycle time of a frame consists of four intervals:
(i) Idle $\left(\mathrm{T}_{\text {idle }}\right)$
(ii) Sensing-Sharing $\left(\mathrm{T}_{\mathrm{ss}}\right)$
(iii) Contention $\left(\mathrm{T}_{\mathrm{ct}}\right)$
(iv) Transmission $\left(\mathrm{T}_{\text {tr }}\right)$

CR idle interval is constant time to indicate the beginning of new cycle and during sensing-sharing interval secondary users sense the primary channels and exchange the sensing results. $\mathrm{T}_{\mathrm{ct}}$ consists of $\mathrm{M}$ contention slots, which enables secondary users to compete with each other to reserve the sensed idle channel to be used for their data transmission. After contention, successful SUs can transmit data frames in parallel on self schedule idle channels during the transmission period and all the SUs are assumed to be synchronized.

In the conventional frame structure, secondary user that employs this scheme ceases data transmission at $T_{\mathrm{ss}}$ slot and performs spectrum sensing for specified units of time and uses the remaining frame duration for contention and data transmission, as shown in fig. 1. Therefore, a tradeoff exists between the duration of spectrum sensing and data transmission. Moreover, this frame structure disrupts the continuous communication in spectrum sharing cognitive radio networks and decreases their throughput by a factor of $\mathrm{T}_{\mathrm{ss}}$ when the primary users are active.

\section{ENHANCED FRAME STRUCTURE}

In proposed MAC protocol the frame structure is modified such that spectrum sensing and data transmission occurs simultaneously which not only reduces the cycle time but also enhance the throughput by eliminating sensing throughputtradeoff as shown in fig. 2. In the FCS, each secondary user senses only fixed number of licensed channels regardless of the traffic load of licensed channels. In the transmission interval, a cognitive radio sends the frame over the sensed idle channel successfully. Data transmission is only possible if transmitting cognitive user has chosen different contention slot in contention interval and a CR sends request (CR-RTS) in contention interval and if the idle channel is available then after short time CR acknowledged with CR-CTS (Clear to Send). In SMC-MAC, it has been proposed that each secondary user randomly chooses a contention slot which reduces the probability of collisions among SUs. We have considered the distributed cognitive radio network and its MAC protocol as in SMC-MAC [13].

\subsection{Fixed Channel Sensing (FCS)}

In FCS, number of sensed channels is fixed regardless of traffic load of primary users. Let us assume that cognitive user can sense only $\mathrm{CH}_{\mathrm{MAX}}$ channels randomly among total $\mathrm{CH}_{\mathrm{T}}$ licensed channels. Here, the $\mathrm{CH}_{\mathrm{MAX}}$ is fixed, therefore it is also called FCS. Then the probability distribution of the number of sensed idle channels $\mathrm{j}$ among $\mathrm{CH}_{\mathrm{MAX}}$ channels by a single cognitive user is [12]:

$p_{j}(j)\left(\frac{\mathrm{CH}_{\text {MAX }} !}{j ! *\left(\mathrm{CH}_{\operatorname{MAX}}-\mathrm{j}\right) !}\right) *\left(1-\mathrm{l}_{\mathrm{p}}\right) \cdot \mathrm{l}_{\mathrm{p}}{ }^{\mathrm{CH}_{\text {MAX }}-\mathrm{j}}, \quad 0 \leq \mathrm{j} \leq \mathrm{CH}_{\mathrm{MAX}}$

Thus the average number of sensed idle channels by a cognitive user is:

$\Delta[\mathrm{J}]=\sum_{\mathrm{j}=0}^{\mathrm{CH}_{\text {MAX }}} \mathrm{j} \cdot \mathrm{p}_{\mathrm{j}}(\mathrm{j})$

Where, $p_{j}(j)$ is from Eq. (1). Then, the probability that an idle channel is sensed among the actual idle channels by a cognitive user is defined as:

$\eta=\frac{\Delta[\mathrm{I}]}{\Delta[\mathrm{J}]}$

As said earlier SUs choose and sense the licensed channels independently, the probability $\mathrm{p}_{\mathrm{s}}(\mathrm{s})$ that an idle channel is sensed by s SUs follows the binomial distribution as:

$\mathrm{p}_{\mathrm{s}}(\mathrm{s})=\left(\frac{\mathrm{CH}_{\mathrm{SU}} !}{\mathrm{s} ! *\left(\mathrm{CH}_{\mathrm{SU}}-\mathrm{s}\right) !}\right) * \eta^{\mathrm{s}}(1-\eta) \cdot \mathrm{l}_{\mathrm{p}}{ }^{\mathrm{CH}_{\mathrm{SU}}-\mathrm{s}}, 0 \leq \mathrm{j} \leq$

$\mathrm{CH}_{\mathrm{SU}}$

From Eq. (3), we can obtain the probability that an idle channel is not sensed by any CHSU number of cognitive users, which is given by: 
$\mathrm{P}_{\text {Fnosensed }}=(1-\eta)^{\mathrm{CH}} \mathrm{su}$

From Eq. (5), the probability that an idle channel is sensed by at least one cognitive user is:

$\mathrm{P}_{\text {Fsensed }}=1-\mathrm{P}_{\text {Fnosense } \mathrm{d}}$

The probability distribution of the number of sensed idle channels $\mathrm{n}$ by $\mathrm{CH}_{\mathrm{SU}}$ cognitive users is determined as:

$\mathrm{p}(\mathrm{n})=\left(\frac{\mathrm{j} !}{\mathrm{n} ! *(\mathrm{j}-\mathrm{n}) !}\right) \mathrm{P}_{\text {Fsensed }} *\left(1-\mathrm{P}_{\text {Fsensed }}\right)^{\mathrm{j}-\mathrm{n}}$

, $0 \leq \mathrm{n} \leq \mathrm{j}$

From Eq. (7), the average number of sensed idle channels by $\mathrm{CH}_{\mathrm{SU}}$ cognitive users is calculated as:

$\Delta[\mathrm{N}]=\sum_{\mathrm{n}=0}^{\mathrm{CH}_{\mathrm{T}}} \mathrm{n} \cdot \mathrm{p}(\mathrm{n})$

The transmission time is calculated as in [9]

$\mathrm{T}_{\text {tr }}$ (conv. $)=\mathrm{T}_{\text {cycle }}-\left(\mathrm{T}_{\text {idle }}+\mathrm{T}_{\mathrm{ss}}+\mathrm{T}_{\text {ct }}\right)$

The transmission time $\mathrm{T}_{\text {tr }}$ per data channel is found by subtracting the idle time $\mathrm{T}_{\text {idle }}$, the sensing-sharing time $\mathrm{T}_{\mathrm{ss}}$, and contention time $\mathrm{T}_{\text {ct }}$ from cycle time $\mathrm{T}_{\text {cycle }}$. These time intervals can be formalized as:

$\mathrm{T}_{\text {idle }}=$ SIFS time $+2 *$ slot time

$\mathrm{T}_{\mathrm{sS}}=\mathrm{CH}_{\mathrm{T}}+2 *$ ss slot time

$\mathrm{T}_{\mathrm{ct}}=\mathrm{M} *$ contention time slot

For proposed scheme as shown in fig. 2, secondary user senses the channels along with data transmission slot which not only reduce $\mathrm{T}_{\text {cycle }}$ but also the throughputs. Spectrum sensing-sharing and data transmission share same slot as we are performing data transmission and spectrum sensingsharing simultaneously. Now $\mathrm{T}_{\text {tr }}$ is written as:

$\mathrm{T}_{\text {tr }}$ (prop. $)=\mathrm{T}_{\text {cycle }}-\left(\mathrm{T}_{\mathrm{idle}}+\mathrm{T}_{\mathrm{ct}}\right)$

\subsection{Throughput Analysis}

In FCS maximum throughput can be given as:

$\mathrm{TH}_{\max }^{\mathrm{FCS}}$ (Prop) $=\frac{\left[\mathrm{T}_{\text {cycle }}-\left(\mathrm{T}_{\text {idle }}+\mathrm{T}_{\text {ct }}\right) * \Delta[\mathrm{N}] * \mathrm{R}\right]}{\mathrm{T}_{\text {cycle }}}$

Where, $\mathrm{R}$ is the data rate per channel and average number of sensed idle channels $\Delta[\mathrm{N}]$ is from Eq. (8). The real throughput of FCS is determined by considering the contention mechanism. Since each successful SU can use CHidle idle channels that all successful s secondary users transmit data frames is $\mathrm{s}^{*}$ CHidle and number of randomly sensed idle channels be $\mathrm{N}$. Therefore number of idle channels used for real throughput is the minimum of $\mathrm{s} . \mathrm{CH}_{\text {idle }}$ and $\mathrm{N}$. Thus real throughput can be expressed as

$\mathrm{TH}_{\text {real }}^{\mathrm{FCS}}(\mathrm{prop})=$

$\frac{\left.\Delta[\min (\mathrm{s} * \text { CHidle } * \mathrm{~N})] \mathrm{T}_{\text {cycle }}-\left(\mathrm{T}_{\text {idle }}+\mathrm{T}_{\text {ct }}\right) \mathrm{R}\right]}{\mathrm{T}_{\text {cycle }}}$

\section{SIMULATION RESULTS}

Figure 3 is showing the relationship between average number of sensed idle channels and Traffic load of primary users. It is observed that the average numbers of sensed idle channels by active secondary users in fixed channel sensing are close to the actual number of idle channels with an increase in the number of channels sensed by SUs. For e.g. when the traffic load of primary user $l p=0.5$, the average number of sensed idle channels with $C H_{M A X}=2,3$ and 4 in FCS are 6.52, 8.03and
8.92 respectively, while the actual number of idle channels is 10.conventional and proposed scheme for FCS

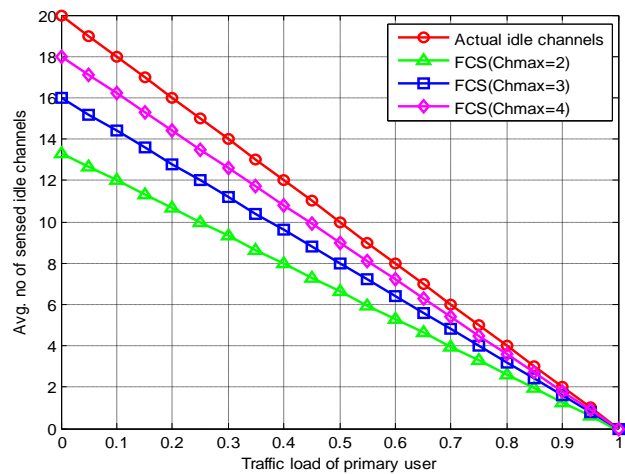

Figure 3: Average number of sensed idle channels by active SUs for different $\mathrm{CH}_{M A X}, \mathrm{CH}_{S U}=10$ and $\mathrm{CH}_{\text {idle }}=2$.

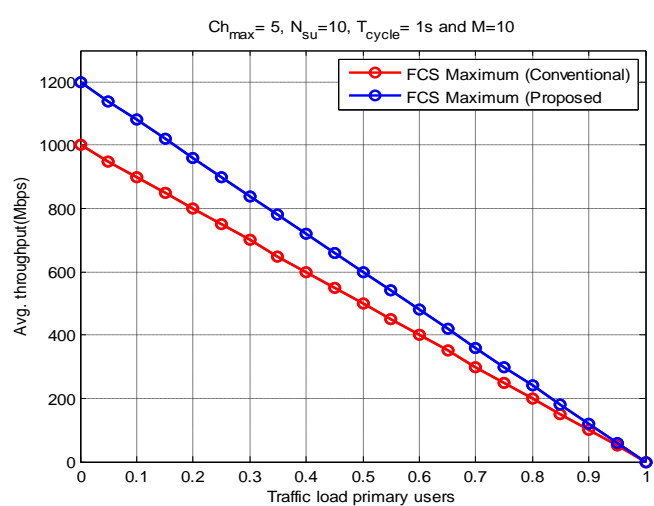

Figure 4: Comparison of maximum throughput of

In Figures 4 and 5 we will compare the maximum and real throughputs of FCS for conventional and proposed shemes. Figure 4 shows the maximum throuput of FCS scheme with respect to primary traffic load. The parameters taken into consideration $M=10, C_{M A X}=5, C H_{S U}=10$ and $T_{C y c l e}=1 \mathrm{sec}$. It could be observe that the average throughput increases significantly from $1000 \mathrm{Mbps}$ to $1200 \mathrm{Mbps}$ when no primary traffic is available on channel. With an increase of primary traffic the throughput start decreasing and become equal to zero(similar to the conventional approach) when all the channels are occupied by primary user (1 Erlang). Similar observations have been made for the real throughput of conventional and proposed scheme for FCS as shown in fig. 5.

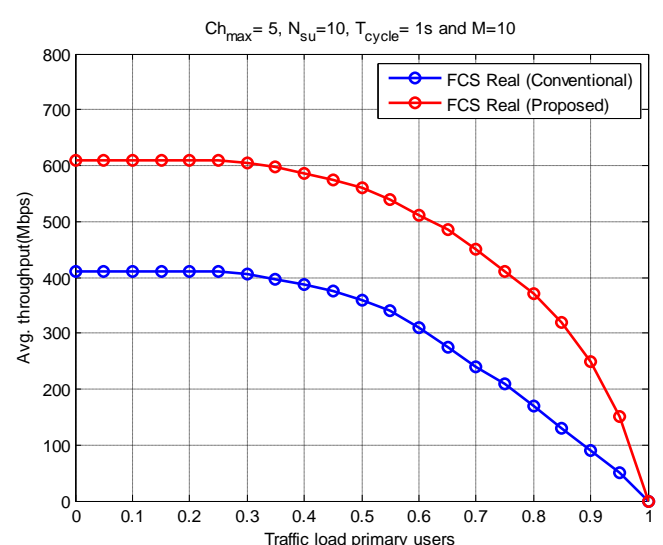

Figure 5: Comparison of real throughput of conventional and proposed scheme for FCS. 


\section{CONCLUSION AND FUTURE SCOPE}

From theoretical and simulated results the inference has been drawn that for the conventional MAC protocol, the time slots given for the sensing and data transmission for the fixed channel sensing were fixed. Significant slot duration was assigned for wide band scanning by cognitive radio. Because of this the transmission period was lesser and therefore cognitive radio throughput was lesser. In this paper, frame structure has been modified such that spectrum sensing and transmission occurs in parallel and therefore transmission time increases significantly that ultimately leads to the higher throughput. The $\mathrm{CR}$ is a new research topic and there is a tremendous scope of increasing throughput of CR network by proposing new MAC layer protocols by designing new frame structure.

\section{REFERENCES}

[1] J. Mitola and J. Maguire, G. Q., "Cognitive Radio: making software radios more personal," IEEE Pers. Communication Magazine, vol. 6, no. 4, pp. 13-18, Aug. 1999

[2] S. Haykin, "Cognitive radio: brain-empowered wireless communications," IEEE Journal on Selected Areas in Communication, vol. 23, pp. 201-220, Feb. 2005.

[3] Indu Bala, Manjit Singh Bhamrah and Ghanshyam Singh, "Analytical Modelling of Ad Hoc Cognitive Radio Environment for Optimum Power Control," International. Journal of Computer Applications, vol. 92, No. 7, pp. 19-22, 2014.

[4] Vanita Rana, Indu Bala and Neelu Jain, "Resource Allocation Models for Cognitive Radio Networks: A Study," International Journal of Computer Applications, vol. 91, No. 12, pp. 51-55, 2014

[5] Rubeena Sethi and Indu Bala, "Performance Evaluation of Energy Detector for cognitive radio network," IOSR journal of Electronics and communication, issue 5, vol. 8 pp 46-51, 2013.
[6] Q. Zhao, L. Tong, A. Swami, and Y. Chen, "Decentralized cognitive MAC for opportunistic spectrum access in ad hoc networks: A POMDP framework," IEEE Journal on Selected Areas of Communication, vol.25, no.3, pp. 589-600, April 2007.

[7] H. Su and X. Zhang, "Opportunistic MAC protocols for cognitive radio based wireless networks," in proceedings of 41st Conference on Information Sciences and Systems, pp.363-368, John Hopkins University, USA, March 2007.

[8] J. Jia, Q. Zhang, and X. Shen, "HC-MAC: A hardwareconstrained cognitive MAC for efficient spectrum management," IEEE Journal on Selected Areas of Commununications, vol.26, no.1, pp.106-117, Jan. 2008.

[9] Shweta Pandit and G. Singh, "Self-Scheduled MACLayer Protocol for Spectrum Sharing in Cognitive Radio Communication," IEEE, 2013.

[10] C. Cordeiro and K. Challapali, "C-MAC: a cognitive MAC protocol for multi-channel wireless networks," in proceedings of 2nd IEEE International Symposium on New Frontiers in Dynamic Spectrum Access Networks (DySPAN), pp. 147-157, April 2007.

[11] S. Lim and J. Lee, "A self-scheduling multi-channel cognitive radio MAC protocol based on cooperative communications," IEICE Transactions on Communications, vol. E94-B, no.6, pp.1657-1668, June 2011.

[12] Ying-Chang Liang, Yong hong Zeng, E. C. Y. Peh and Anh Tuan Hoang, "Sensing Throughput Tradeoff for Cognitive Radio Networks," IEEE Transactions on Wireless Communications, vol.7, no.4, pp.1326-1337, April 2008.

[13] Xin Kang, "Sensing-based Spectrum Sharing in Cognitive Radio Networks," IEEE Transactions on Vehicular Technology, issue 8, vol. 58, 2009 